\title{
Considering on charge density along return stroke lightning channel
}

\begin{abstract}
Prediction of return stroke current wave shape along lightning channel is an important subject in evaluating the electromagnetic fields and induced overvoltage due to lightning channel at observation point and power line. This paper reviews the different return stroke current models, with the engineering current models is selected as the leading model, and evaluates the their current behaviors along the lightning channel. A comprehensive result on the charge density for each model will then be presented to conclude the findings.
\end{abstract}

Keyword: Return stroke current; Lightning channel; Induced overvoltage; Charge density 